
PSOR MEIO DA CLARIFICAÇÃO COM TANFLOC

\title{
GLYPHOSATE AND TURBIDITY REMOVAL IN WATER CONDITIONS BY CLARIFICATION WITH TANFLOC
}

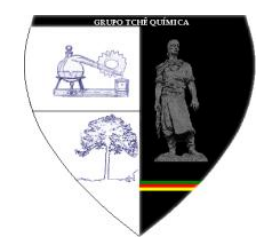

\author{
SILVA, Julia Thais Bueno ${ }^{1 *}$; ROCHA, Ketley Costa $^{2}$; CUBA, Renata Medici Frayne ${ }^{3}$; \\ ${ }^{1,2}$ Pontifícia Universidade Católica de Goiás, Escola de Engenharia, \\ Av Universitária, 1.440, Setor Universitário, cep 74605-010, Goiânia - GO, Brasil
}

(fone: +55 62 3946-1000)

${ }^{3}$ Universidade Federal de Goiás, Departamento de Engenharia, Avenida Esperança, s/n, cep 74690-900, Goiânia - GO, Brasil

(fone: +5562 3521-1000)

${ }^{*}$ Autor correspondente

e-mail: juliathaisbueno@gmail.com

Received 08 March 2018; received in revised form 1st July 2018; accepted 11 July 2018

\section{RESUMO}

A partir do progresso no setor agrícola, ocasionou-se um indevido domínio sobre o uso de defensores agrícolas, herbicidas, inseticidas, que afetam negativamente o meio ambiente até os dias de hoje. Portanto, o presente trabalho tem por objetivo avaliar a eficiência do coagulante natural Tanfloc, na etapa de clarificação quanto à remoção do herbicida glifosato e do parâmetro turbidez empregando jar test sob diferentes condições de $\mathrm{pH}$ e concentrações de coagulante. Como resultado, foi obtido que para a faixa de $\mathrm{pH}$ 5-5,5 o Tanfloc se mostrou bastante eficiente, com uma porcentagem de remoção de glifosato aproximadamente $98 \%$ utilizando concentrações menores e remoção de turbidez 21,69\%. Já para a faixa de pH entre 6,8-7,3 não apresentou resultados proveitosos, sendo que para algumas concentrações não foram possível detectar as remoções do herbicida. Nas concentrações em que foram detectadas remoções, a porcentagem de remoção de glifosato obteve-se uma média de aproximadamente $89 \%$ e uma média de $20,24 \%$ de remoção de turbidez. Apesar do coagulante natural não ter se mostrado tão eficiente na faixa de $\mathrm{pH}$ neutra, o produto pode ser considerado alternativo no tratamento quanto ao uso de coagulantes metálicos, componentes que produzem lodo com características químicas e que podem trazer consequências ambientalmente negativas.

Palavras-chave: Coagulante natural, eficiência, concentração, $\mathrm{pH}$.

\section{ABSTRACT}

With the progress in the agriculture sector, improper domains of pesticides, herbicides, and insecticides have grown, which have been negatively affected the environment until the present day. Therefore, the present work has as objective to evaluate the efficiency of the natural coagulant Tanfloc in the clarification stage with respect the herbicide glyphosate removal and the turbidity parameter by using jar test with different $\mathrm{pH}$ conditions and coagulant concentration. As a result, was obtained that for the $\mathrm{pH}$ range of 5-5.5 the natural coagulant has shown more efficient, with a glyphosate percentage removal of approximately $98.0 \%$ using low concentrations and turbidity removal of $21.69 \%$. On the other hand, for the $\mathrm{pH}$ range of 6.8 to 7.3 , the coagulant has not shown profitable results, considering that, for some concentrations, it was not possible to detect the herbicide removal. In the concentrations that were detected removal, the average glyphosate percentage removal was approximately $89 \%$ and an average of $20.24 \%$ of turbidity removal. In summary, although the natural coagulant has not shown remarkably efficient in the neutral $\mathrm{pH}$ range, the product may be considered an alternative device in water treatment with the respect of the use of metallic coagulants, which produce sludge with chemistry characteristics that may negatively affect the environment.

Keywords: Natural coagulant, efficiency concentration, $\mathrm{pH}$. 


\section{INTRODUÇÃO}

A modernização das técnicas utilizadas na agricultura que permitiram aumento considerável na produção a partir de 1970, além dos benefícios, trouxe inúmeros impactos ambientais negativos (OCTAVIANO, 2010) ocasionados, principalmente, pela inadequada gestão das atividades que suportam o setor.

Entre essas atividades destaca-se o uso de agroquímicos que, nos últimos 40 anos, aumentou $700 \%$ enquanto que o aumento da área agrícola plantada foi de $78 \%$, valor esse que equivale a consumo médio anual superior a 300 mil toneladas de produtos comerciais (SPADOTTO; GOMES, s/d).

Entre os agrotóxicos destaca-se a classe dos herbicidas, utilizados para destruir ou inibir o crescimento de ervas daninhas. Nessa classe o glifosato foi o composto mais comercializado $(62,4 \%)$ no ano de 2012 totalizando mais de 180 mil toneladas (IBGE, 2015).

O glifosato, quimicamente classificado como organofosforado, é um herbicida pósemergente altamente efetivo e não seletivo que apresenta uma alta solubilidade em água (PEREIRA, 2010) e quando adsorvido à partículas do solo ou sedimentos aquáticos este apresenta elevada persistência biológica e fotoquímica (AMARANTE JUNIOR; SANTOS, 2002).

Com relação a sua toxicidade, as conclusões são bastantes divergentes, pois enquanto trabalhos como o realizado por Williams, Kroes e Munro (2000) concluíram, após ampla revisão da literatura, que o glifosato não apresenta riscos potenciais à saúde humana e ambiente. Estudos mais recentes, como realizado por Paganelli et al. (2010), tem demonstrado que as formulações comerciais a base de glifosato tem efeitos tóxicos potenciais tanto para organismos aquáticos quanto a saúde.

Lajmanovich et al. (2010) e Yadav et al. (2013), ao avaliarem o efeito de formulações comerciais de glifosato em diferentes espécies de anfíbios, verificaram que, mesmo em concentrações ambientalmente esperadas, esses produtos apresentaram efeito letal sobre os organismos testados. Situação essa que pode trazer consequências negativas para as espécies estudadas.

Quanto à saúde humana, segundo estudos feitos pela Agência Nacional de Vigilância Sanitária (Anvisa) em 2008, foi constatado que o glifosato indicava potencial cancerígeno (BRASIL, 2008), fato este corroborado em 2015 pela Agência Internacional do Câncer (IARC) que incluiu o herbicida glifosato na categoria de composto com prováveis efeitos carcinogênicos nos seres humanos (WHO, 2015). No mesmo ano o Instituto Nacional do Câncer (INCA) apoiou a decisão do IARC com a publicação de um documento alertando sobre os possíveis efeitos do glifosato na saúde humana (INCA, 2015).

Essa situação é preocupante, pois embora a aplicação do glifosato seja realizada primordialmente no solo, diferentes estudos tem demonstrado sua presença em ambientes aquáticos como, por exemplo, Suíça (HANKE et al., 2010), Canadá (VAN STEMPVOORT et al., 2014), México (RUIZ-TOLEDO et al., 2014) entre outros, sendo que no trabalho realizado no México foi apurado que as águas subterrâneas e superficiais teriam contaminação por glifosato, incluindo áreas naturais protegidas, amostras de água de sistema fluvial e de drenagem urbana.

Por essas situações a legislação brasileira por meio da Portaria 2.914 de 2011 do Ministério da Saúde estabelece como padrão de potabilidade para o glifosato mais seu principal metabólito de degradação, o ácido aminometilfosfônico (AMPA) máxima concentração de $500 \mu^{-1} \mathrm{~L}^{-1}$ (BRASIL, 2011) o que leva às estações de tratamento de água a adotarem sistemas de tratamento que removam esse composto do meio líquido.

Na literatura são apresentados diferentes métodos para a remoção de glifosato em meio líquido tais como, nanofiltração (SAITÚA; GIANNINI; PADILLA, 2012) ozonização, fotólise e fotocatálise (ASSALIN et al., 2009), e principalmente adsorção (CARNEIRO et al., 2015), tecnologias estas, que não são utilizadas nos sistemas de tratamento convencionais, principalmente devido aos custos envolvidos nesses processos (HU; ZHAO; SOROHAN, 2011).

Desta forma, informações sobre a capacidade de remoção do glifosato nas unidades que compõe os sistemas convencionais de tratamento de água se tornam relevantes do ponto de vista tanto de saúde pública quanto de 
custos envolvidos no tratamento.

No Brasil, os sistemas de tratamento convencionais consistem, basicamente das etapas de clarificação, desinfecção, fluoretação e correção de $\mathrm{pH}$.

Segundo Cardoso (2007 apud VAZ et al., 2010), os dois termos, coagulação e floculação podem ser utilizados como sinônimos e consistem na remoção de substâncias coloidais (materiais em suspensão finamente divididos ou dissolvidos), por meio da desestabilização de cargas superficiais negativas das partículas que diminui a repulsão entre elas seguida pela agregação das mesmas formando flocos mais densos e com maiores velocidades de sedimentação (SCHOENHALS; SENA; JOSÉ, 2006; VAZ et al., 2010).

A natureza dos produtos formados nesse processo é diretamente afetado pelo $\mathrm{pH}$ de origem da água, alcalinidade, temperatura, outras partículas, matéria orgânica, tipo e dosagem do coagulante (AMIRTHARAJAH; O'MELIA, 1990).

Coagulantes inorgânicos como sulfato de alumínio $\left(\mathrm{Al}_{2}\left(\mathrm{SO}_{4}\right)_{3}\right)$ e cloreto férrico $\left(\mathrm{FeCl}_{3}\right)$ e polímeros auxiliares de coagulação, como por exemplo, os polímeros à base de poliacrilamida (PAM), geralmente são os mais utilizados devido a razões econômicas (SANTOS et al., 2014) e comprovação da eficácia por meio de diferentes estudos (GUEDES et al., 2004; CAMPOS; DI BERNARDO; VIEIRA, 2005).

Porém, coagulantes metálicos, não são considerados ambientalmente eficazes, devido ao fato de que os lodos produzidos por esses sais disponibilizam íons solúveis que podem comprometer a saúde humana, como é o caso de sais de alumínio, que dependendo da dosagem de alumínio acumulada no organismo, pode ser tóxico causando grandes danos à saúde como doenças de coordenação motora e demência, Alzheimer e mal de Parkinson. (FERREIRA et al., 2008). Além disso, o lodo produzido tem característica inorgânica de difícil manuseio e alto teor de umidade (VAZ et al., 2010).

Devido a isso, coagulantes de origem natural e ambientalmente mais compatíveis vem sendo bastante empregados no tratamento de águas, como é o caso do Tanfloc. Além de ser um produto natural, renovável e biodegradável também mostra-se economicamente viável, já que requer dosagens menores (VAZ et al., 2010).

O Tanfloc é obtido a partir de processos de lixiviação da casca de arbóreas, como a da Acácia negra (Acácia mearnsii de wild), é constituído por tanato quartenário de amônio (VAZ et al., 2010) cuja estrutura molecular encontra-se apresentada na Figura 1.

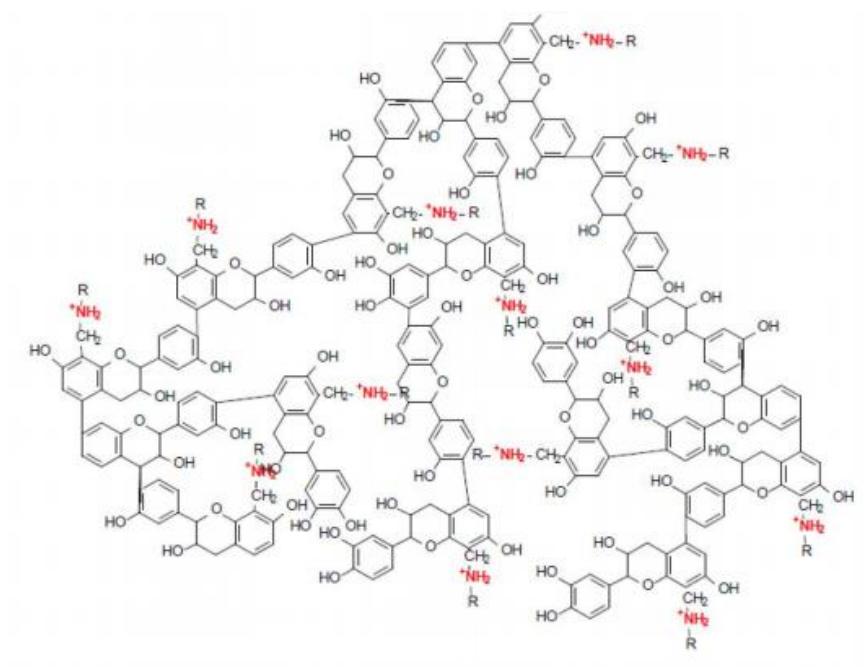

Figura 1. Estrutura do coagulante Tanfloc da empresa TANAC, obtido a partir do tanino extraído da Acácia Negra.

As propriedades coagulantes dos taninos se dá em função da desestabilização dos coloides por meio da eliminação da camada de solvatação, diminuindo o potencial zeta durante o processo de coagulação e, assim, permitindo a formação de flocos (SILVA, 1999).

Comprovados por estudos feitos por Vaz et al (2010) e Bongiovani et al (2010), o Tanfloc apresentou resultados positivos na remoção de cor e turbidez e possui benefícios de saúde pública e de preservação ambiental.

Desta forma, baseando-se na estrutura molecular do Tanfloc e na capacidade de o glifosato fazer ligações de hidrogênio em $\mathrm{pHs}$ ácido ou neutro, com material húmico (MAZZEI; PICCOLO, 2012), acredita-se que a remoção de glifosato em suas formulações comerciais poderia ser representativa durante a coagulação/floculação utilizando o Tanfloc como agente coagulante na remoção de turbidez.

Desta forma, o objetivo fundamental dessa pesquisa foi avaliar a eficiência do coagulante natural Tanfloc na remoção de glifosato e turbidez sob diferentes condições de 
concentração de coagulante e valores de pH.

\section{MATERIAIS E MÉTODOS}

Para a verificação da remoção de glifosato e turbidez, foram realizados ensaios com Tanfloc da marca TANAC S.A. nas concentrações de $5,5 \mathrm{mgL}^{-1}, 11 \mathrm{mgL}^{-1}, 16 \mathrm{mgL}^{-1}$, $22 \mathrm{mgL}^{-1}, 33 \mathrm{mgL}^{-1}$ e $44 \mathrm{mgL}^{-1}$ e duas faixas de $\mathrm{pH}$ sendo 5,0 a 5,5 e 6,8 a 7,3 .

A concentração de glifosato utilizada nos ensaios foi de $8 \mathrm{mg} . \mathrm{L}^{-1}$ escolhida levando-se em consideração o limite de detecção do método de análise proposto por Tzaskos et al. (2012), e obtida por meio de diluições de solução estoque com concentração de $5 \mathrm{~g} \cdot \mathrm{L}^{-1}$ preparada a partir do produto comercial à base de sal de Di-amônio de Glifosato 445g. $\mathrm{L}^{-1}$ (370g. $\mathrm{L}^{-1}$ equivalente de ácido).

A água utilizada para os ensaios foi água bruta do manancial João Leite coletada na Estação de Tratamento de Água (ETA) Jaime Câmara da SANEAGO, cuja turbidez foi mantida entre 20 a 30 NTU utilizando-se lodo do decantador da ETA, quando necessário.

Os ensaios foram realizados em equipamento de jar test (Figura 2) utilizando seis jarros com capacidade de 2 litros cada, porém, o volume de água analisado foi de $1 \mathrm{~L}$ por jarro. A correção do $\mathrm{pH}$ e turbidez nos valores desejados era feita em $6 \mathrm{~L}$ de água que posteriormente eram divididos nos jarros.

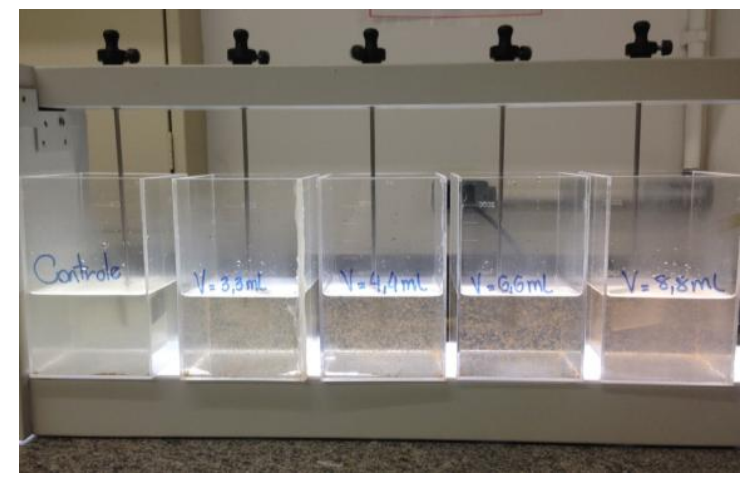

Figura 2: Equipamento jar test.

As condições de operação do equipamento foram adotadas de acordo com as condições utilizadas na ETA Jaime Câmara e se encontram apresentadas no Quadro 1.

\begin{tabular}{|c|c|c|c|c|}
\hline $\begin{array}{c}\text { Rotação } \\
\text { (rpm) }\end{array}$ & 260 & 190 & 30 & Decantação \\
\hline Tempo & $30^{\prime \prime}$ & $1^{\prime} 20^{\prime \prime}$ & $20^{\prime}$ & $5^{\prime}$ \\
\hline
\end{tabular}

Quadro 1: Condições de operação do equipamento.

Após as etapas de coagulação, floculação e decantação o pH, turbidez e concentração de glifosato eram medidos. Para a análise de glifosato segundo método proposto por Bhaskara e Nagaraja (2006) a amostra de água decantada era previamente filtrada em membrana de celulose com diâmetro de poro de 0,45 $\mu \mathrm{m}$ e o $\mathrm{pH}$ corrigido para o intervalo de 6,7 a 7,3.

Foi utilizado o programa Statistica para a construção dos diagramas de remoção de glifosato e turbidez a fim de realizar a análises estatísticas a partir dos resultados obtidos.

\section{RESULTADOS E DISCUSSÃO:}

Os resultados de remoção do glifosato com o coagulante orgânico Tanfloc em ambas as faixas de pHs, encontram-se apresentados na Figura 3.

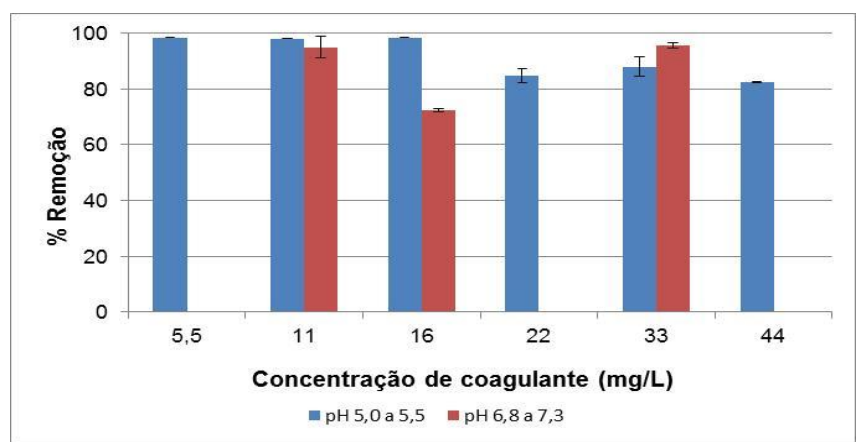

Figura 3: Comparação da eficiência de remoção do glifosato utilizando Tanfloc como coagulante nas faixas de $\mathrm{pH}$ 5,0 a 5,5 e 6,8 a 7,3.

Ao analisar os dados de remoção do glifosato com o auxílio do coagulante orgânico Tanfloc verifica-se que para a faixa de $\mathrm{pH}$ entre 5,0 a 5,5 a remoção foi de $98,0 \%$, sendo que para as concentrações superiores a $16 \mathrm{mg} \cdot \mathrm{L}^{-1}$ teve queda de aproximadamente $13 \%$ na eficiência que ficou em torno de $85 \% \pm 2,84 \%$.

Já, com relação à faixa de $\mathrm{pH}$ 6,8 a 7,3 a eficiência de remoção não apresentou 
comportamento padrão, inclusive não sendo possível detectar remoção de glifosato nas concentrações de $5 \mathrm{mg} \cdot \mathrm{L}^{-1}$, 22mg. $\mathrm{L}^{-1}$ e $44 \mathrm{mg} \cdot \mathrm{L}^{-1}$ de Tanfloc.

Com relação à remoção de turbidez, entre ambos os intervalos de pHs não houve diferença significativa, sendo $21,69 \% \pm 1,18 \% \quad e$ $20,23 \% \pm 1,77 \%$, para as faixas ácida e neutra, respectivamente, o que leva a hipótese de que a diferença de remoção do glifosato comparando ambos os pHs não foi associado à remoção de turbidez.

Entretanto, ao comparar a eficiência de remoção de turbidez com a remoção do glifosato em uma mesma faixa de $\mathrm{pH}$ para as diferentes concentrações de coagulante, especificamente a faixa ácida, os resultados indicam uma relação direta entre ambos os parâmetros, sendo que o aumento da concentração de coagulante afetou negativamente a remoção de ambos.

No caso do intervalo de $\mathrm{pH}$ entre 5,0 a 5,5 para as concentrações de $5,5 \mathrm{mg} \cdot \mathrm{L}^{-1}$ a $16 \mathrm{mg} . \mathrm{L}^{-1}$ a remoção média de turbidez e glifosato foi, respectivamente, $22,71 \% \pm 0,59 \%$ e $98,0 \%$, enquanto que para as concentrações $22 \mathrm{mg} \cdot \mathrm{L}^{-1}$, $33 \mathrm{mg} \cdot \mathrm{L}^{-1}$ e $44 \mathrm{mg} \cdot \mathrm{L}^{-1}$ a remoção média da turbidez foi de $20,68 \% \pm 0,20 \%$ e $85 \% \pm 2,83$ para o glifosato.

Nota-se que com as maiores concentrações de coagulante a eficiência de remoção de turbidez e glifosato diminuíram em $8,9 \%$ e $13,26 \%$, respectivamente, quando comparadas às eficiências obtidas com as menores concentrações de coagulante.

$\mathrm{Na}$ Figura 4 são apresentados gráficos nos quais é possível avaliar o efeito conjunto da alteração do $\mathrm{pH}$ e concentração de coagulante na remoção do glifosato e turbidez.

Embora a atuação do Tanfloc seja efetiva em uma faixa de $\mathrm{pH}$ entre 4,5 a 8,0 (CORAL; BERGAMASCO; BASSETTIC, 2009), foi possível verificar na Figura 3 que tanto para o glifosato quanto para a turbidez a eficiência de remoção foi maior para as condições de menor $\mathrm{pH}$ e concentração de coagulante.

Oliveira e Cruz (2013) e Hameed et al. (2016) explicam que diferente dos coagulantes tradicionalmente utilizados que necessitam de ajuste do $\mathrm{pH}$ para seu melhor rendimento, o processo de coagulação ocorre diretamente pela fração catiônica natural presente em suas moléculas.

Desta forma, ao analisar a estrutura molecular do Tanfloc (Figura 1), nota-se que pHs ácidos favoreceriam a protonação da molécula o que poderia favorecer a remoção do glifosato por interações eletrostáticas, visto que este composto pode ser considerado polar (GALLI; MONTEZUMA, 2005) e ser capaz de fazer ligações de hidrogênio em pHs ácido ou neutro, com material húmico (MAZZEI; PICCOLO, 2012).

Beltran-Heredia et al. (2011) ao avaliarem a capacidade do Tanfloc na remoção do corante Alizarina verificou que em $\mathrm{pH} \mathrm{4,0}$ a remoção foi de aproximadamente $100 \%$ enquanto que em $\mathrm{pH}$ 9,0 a eficiência caiu para $25 \%$. Os autores explicam que a essa menor eficiência pode estar associada à desnaturação da estrutura polifenólica devido à interação dos ânions hidróxidos com a estrutura aromática da molécula.

Com relação à diminuição da eficiência de remoção de turbidez e glifosato com o aumento da concentração de coagulante, outros autores como Vaz et al. (2010) e Ströher et al. (2013) também verificaram esse fenômeno com relação à remoção de turbidez enquanto que Barrado-Moreno, Beltrán-Heredia e MartínGallardo (2016) na remoção de algas, sendo que, neste último os autores associaram o excesso de coagulante com a desestabilização das cargas superficiais das células 0 que influenciou diretamente no processo de coagulação/floculação das mesmas.

Nesse sentido, levando-se em consideração que a remoção tanto do glifosato quanto da turbidez sejam mediante processos eletrostáticos 0 excesso de coagulante no sistema pode ter atuado da mesma forma. 


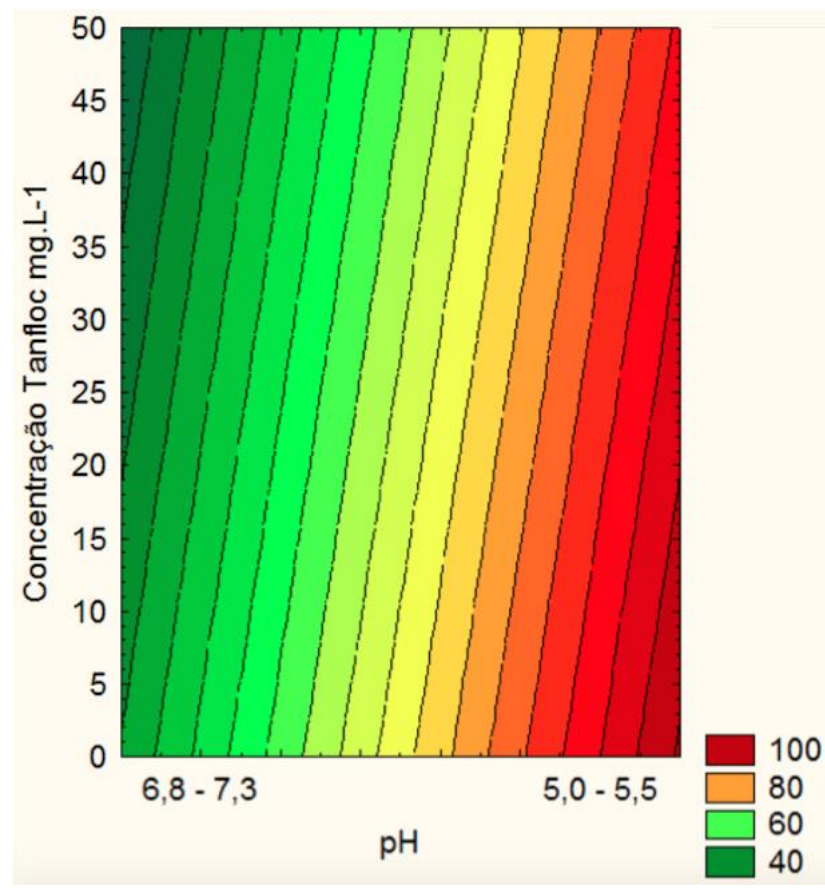

$3 \mathrm{~A})$

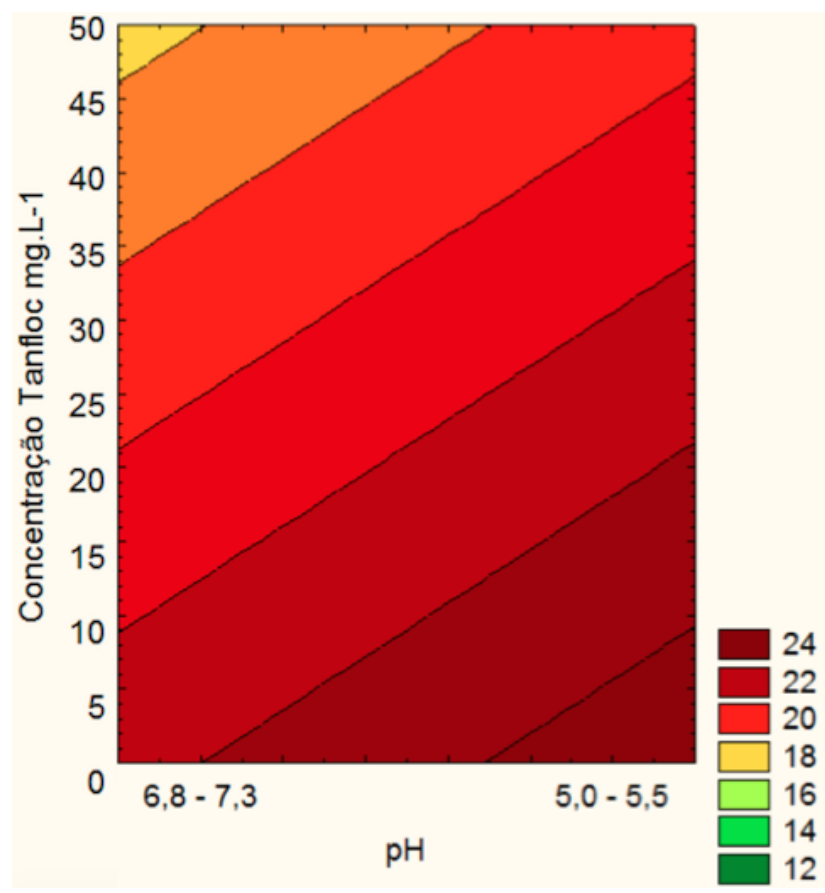

3B)

Figura 4: Efeito conjunto da alteração do $\mathrm{pH}$ e concentração de coagulante na remoção de glifosato ( $3 A$ ) e turbidez (3B).

\section{CONCLUSÕES:}

A maior eficiência de remoção do glifosato foi de $98,0 \%$ e a maior média de eficiência de remoção de turbidez foi de $22,71 \% \pm 0,59 \%$, ambos obtidos na utilização da faixa de $\mathrm{pH}$ de 5,0 a 5,5 e nas concentrações de
5,5; 11 e $16 \mathrm{mgL}^{-1}$. Ou seja, o coagulante natural Tanfloc se mostrou mais eficiente em águas de $\mathrm{pH}$ mais ácido e utilizando concentrações de menores de coagulante.

Além disso, é válido lembrar que coagulantes metálicos requerem uma quantidade maior no uso, assim encarecendo o custo do sistema. $\mathrm{E}$, pelo fato de serem componentes químicos são capazes de produzirem lodo com características químicas de difícil disposição final.

Por isso, aconselha-se o uso de coagulantes naturais, de natureza biodegradável, como é o caso do Tanfloc, que mesmo em concentrações baixas obteve eficácia chegando a uma porcentagem de remoção de glifosato próxima de $100 \%$, e porcentagens de remoção de turbidez admissíveis.

\section{AGRADECIMENTOS:}

Agradecimentos a Saneamento de Goiás S.A. por disponibilizar toda a água utilizada neste estudo.

\section{REFERÊNCIAS:}

1. AMARANTE JUNIOR, O. P.; SANTOS T. C. R. dos. Glifosato: Propriedades, toxicidade, usos e legislação. Quím. Nova, v. 25, n. 4, p. 589-593, 2002.

2. AMIRTHARAJAH, A.; O'MELIA, C. R.. Coagulation processes: Destabilization, mixing and flocculation. Water quality and treatment. 1 ed. Toronto: Ed. McGraw-Hill, 1990. 220 p.

3. ASSALIN, M. R.; MORAES, S. G. de; QUEIROZ, S. C. N.; FERRACINI, V. L.; DURAN, N.. Studies on degradation of glyphosate by several oxidative chemical processes: Ozonation, photolysis and heterogeneous photocatalysis. Journal of Environmental Science and Health, Part B: Pesticides, Food Contaminants, and Agricultural Wastes, London, 45:1, p. 89-94, 2009.

4. BARRADO-MORENO, M. M.; BELTRÁNHEREDIA, J.; MARTÍN-GALLARDO, J.. Microalgal removal with natural coagulants. Phycologia, v. 55, n. 6, p. 688-696, 2016.

5. BELTRAN-HEREDIA, J.; SANCHEZ-MARTIN, J.; MARTIN-SANCHEZ, C.. Remediation of Dye- 
Polluted Solutions by a New Tannin-Based Coagulant Industrial. Engineering Chemistry Research, v. 50, n. 2, p. 686-693, 2011.

6. BHASKARA, B. L.; NAGARAJA, P.. Direct sensitive spectrophotometric determination of glyphosate by using ninhydrin as a chromogenic reagent in formulations and environmental water samples. Helvetica Chimica Acta, v. 89, p. 2686-2693, 2006.

7. BONGIOVANI, M. C.; KONRADT-MORAES, L. C.; BERGAMASCO, R.; LOURENÇO, B. S. S.; TAVARES, C. R. G.. Os benefícios da utilização de coagulantes naturais para obtenção de água potável. Acta Scientiarum. Technolofy, Maringá, v. 32, n. 2, p. 167-170, 2010.

8. BRASIL. Ministério da Saúde. Agencia Nacional de Vigilância Sanitária. Resolução RDC n ${ }^{\circ} 10$ de 22 de Fevereiro de 2008.

9. BRASIL. Portaria no 2.914 de 12 de Dezembro de 2011. Dispõe sobre os procedimentos de controle e de vigilância da qualidade da água para consumo humano e seu padrão de potabilidade. Brasília, 12 dez 2011.

10. CAMPOS, S. X.; DI BERNARDO, L.; VIEIRA, E. M.. Influência das características das substâncias húmicas na eficiência da coagulação com sulfato de alumínio. Eng. Sanit. Ambient. v. 10, n. 3, p. 194-199, 2005.

11. CARNEIRO, R. T. A.; TAKETA, T. B.; GOMES NETO, R. J.; OLIVEIRA, J. L.; CAMPOS, E. V. R.; DE MORAES, M. A.; DA SILVA, C. M. G.; BEPPU, M. M.; FRACETO, L. F.. Removal of glyphosate herbicide from water using biopolymer membranes. Journal of Environmental Management, v. 151, p. 353360, 2015.

12. CORAL, R. L. A.; BERGAMASCO, R.; BASSETTI, F. J.. Estudo da Viabilidade de Utilização do Polímero Natural (TANFLOC) em Substituição ao Sulfato de Alumínio no Tratamento de Águas para Consumo. 2nd International Workshop | Advances in Cleaner Production, São Paulo, Brazil, 2009, 9p.

13. FERREIRA, P. C.; PIAI, K. A.; TAKAYANAGUI, A. M. M.; SEGURA-MUÑOZ, S. I.. Aluminium as a risk factor for Alzheimer's disease. Revista Latino-Americana de Enfermagem, v. 16, n. 1, p. 151-157, 2008.
14. GALLI, A. J. B.; MONTEZUMA, M. C.. Alguns aspectos da utilização do herbicida glifosato na agricultura. ACADCOM Gráfica e Editora Ltda, 2005.

15. GUEDES, C. D.; PEREIRA, J. G.; DE LENA, J. C.; PAIVA, J. F. de. Coagulação/Floculação de suspensões ricas em óxidos de ferro por sulfato de alumínio. Quím. Nova, v. 27, n. 5, p. 715-719, 2004.

16. HAMEED, Y. T.; IDRIS, A.; HUSSAIN, S. A.; ABDULLAH, N.. A tannin-based agent for coagulation and flocculation of municipal wastewater: Chemical composition, performance assessment compared to Polyaluminum chloride, and application in a pilot plant. Journal of Environmental Management, 2016.

17. HANKE, I.; WITTMER, I.; BISCHOFBERGER, S.; STAMM, C.; SINGER, $\mathrm{H}$.. Relevance of urban glyphosate use for surface waterquality. Chemosphere, v. 81, p. 422-429, 2010.

18. HU, Y. S.; ZHAO, Y. Q.; SOROHAN, B.. Removal of glyphosate from aqueous environment by adsorption using water industrial residual. Desalination, v. 271, n. 1-3, p. 150-156, 2011.

19. IBGE. Indicadores de Desenvolvimento Sustentável: Brasil 2015. Rio de Janeiro: IBGE, 2015.

20. Instituto Nacional de Câncer José de Alencar Gomes da Silva (INCA). Posicionamento do Instituto Nacional de Câncer José Alencar Gomes da Silva acerca dos agrotóxicos. (2015). Disponível em: http://www1.inca.gov.br/inca/Arquivos/comunicac ao/posicionamento do inca sobre os agrotoxic os 06 abr 15.pdf. Acessado em 12 mai 2017.

21. LAJMANOVICH, R. C.; PELTZER, P. M.; CABAGNA, M. C.; ATTADEMO, A. M.; JUNGES, C. M.. Toxicity of four herbicide formulations with glyphosate on Rhinella arenarum (Anura: bufonidae) tadpoles: B-esterases and gluthione S-transferase inhibitors. Arch. Environ. Contam. Toxicol., 2011.

22. MAZZEI, P.; PICCOLO, A.. Quantitative evaluation of noncovalent interactions between glyphosate and dissolved humic substances by 
NMR spectroscopy. Environ. Sci. Technol., v. 46, p. 5939-5946, 2012.

23. OCTAVIANO, C.. Muito tecnologia: os impactos da Verde. Com Ciência, v. 120, 2010.

além da Revolução

24. OLIVEIRA, I. M. de; CRUZ, V... Tannin treated water for use in the emulsion polymerization of SBR. Polímeros Ciência e Tecnologia, v. 23, n. 3, p. 326-330, 2013.

25. PAGANELLI, A.; GNAZZO, V.; ACOSTA, H.; LÓPEZ, S. L.; CARRASCO, A. E.. Glyphosate based herbicides produce teratogenic effects on vertebrates by impairing retinoic acid signaling. Chem. Res. Toxicol, v. 23, p. 15861595, 2010.

26. PEREIRA, J.. Remoção de pesticidas em água utilizando adsorventes a base de borra de café. 2010. Tese (Doutorado em Química). Universidade Federal de Goiás, Goiânia.

27. RUIZ-TOLEDO, J.; CASTRO, R.; RIVEROPÉREZ, N.; BELLO-MENDOZA, R.; SÁNCHEZ, $D$.. Occurrence of glyphosate in water bodies derived from intensive agriculture in a tropical region of Southern Mexico. Bulletin of Environmental Contamination and Toxicology, v. 93, n. 3, p. 289-293, 2014.

28. SAITÚA, H.; GIANNINI, F.; PADILLA, A. P.. Drinking water obtaining by nanofiltration from waters contaminated with glyphosate formulations: Process evaluation by means of toxicity tests and studies on operating parameters. Journal of Hazardous Materials, v. 227-228, p. 204-210, 2012.

29. SANTOS, F.S.; OLIVEIRA, S. M. de; VENEU, D. M.; CAMMAROTA, M. C.; YOKOYAMA, L.. Avaliação da eficiência do processo de coagulação/floculação aplicado ao tratamento primário de efluente da indústria petroquímica. Engevista, v. 16, n. 4, p. 404-413, 2014.

30. SCHOENHALS, M.; SENA, R. F.; JOSÉ, H. J.. Avaliação da eficiência do processo de coagulação/flotação aplicado como tratamento primário de efluentes de abatedouro de frangos. Engenharia Ambiental: Espirito Santo do Pinhal, v. 3, n. 2, p. 005-024, 2006.

31. SILVA, T. S. S. de. Estudo de tratabilidade físico-química com uso de taninos vegetais em água de abastecimento e esgoto. 1999. Dissertação (Mestrado) Fundação Oswaldo Cruz, Escola Nacional de Saúde Pública.

32. SPADOTTO, C.A.; GOMES, M.A.F.. Agrotóxicos no Brasil. EMBRAPA, s/d.

33. STRÖHER, A. P.; MENEZES, M. L. de; PEREIRA, N. C.; BERGAMASCO, R.. Utilização de coagulantes naturais no tratamento de efluente proveniente de lavagem de jeans. Engevista, v. 15, n. 3. p. 255-260, 2013.

34. TZASKOS, D. F.; MARCOVIEZ, C.; DIAS, N. M. P.; ROSSO, N. D.. Development of sampling for quantification of glyphosate in natural waters. Ciênc. Agrotec: Lavras, v. 36, n. 4, p. 399-405, 2012.

35. VAN STEMPVOORT, D. R.; ROY, J. W.; BROWN, S. J.; BICKERTON, G.. Residues of the herbicide glyphosate in riparian groundwater in urban catchments. Chemosphere, v. 95, p. 455, 2014.

36. VAZ, L. G. L.; KLEN, M. R. F.; VEIT, M. T.; SILVA, E. A. da; BARBIERO, T. A.; BERGAMASCO, R.. Avaliação da eficiência de diferentes agentes coagulantes na remoção de cor e turbidez em efluente de galvanoplastia. Eclética química: São Paulo, v. 35 , n. 4, p. 45 54, 2010.

37. WILLIAMS, G. M.; KROES, R.; MUNRO, I. C. Safety evaluation and risk assessment of the herbicide roundup and its active ingredient, glyphosate, for humans. Regulatory Toxicology Pharmacology, 31, p. 117-165, 2000.

38. World Health Organization (WHO) / International Agency for Research on Cancer (IARC). Monographs Volume 112: evaluation of five organophosphate insecticides and herbicides (2015)

Disponível em: https://www.iarc.fr/en/mediacentre/iarcnews/pdf/MonographVolume112.pdf. Acessado em 12 mai 2017.

39. YADAV, S. S.; GIRI, S.; SINGHA, U.; BORO, F.; GIRI, A.. Toxic and genotoxic effects of Roundup on tadpoles of the Indian skittering frog (Euflictis cyanophlyctis) in the presence and absence of predator stress. Aquat. Toxicol. p. 123-133, 
PERIÓDICO TCHÊ QUÍMICA • www.periodico.tchequimica.com • Vol. 15 N. 30.

• ISSN 1806-0374 (impresso) • ISSN 1806-9827 (CD-ROM) • ISSN 2179-0302 (meio eletrônico)

(C) 2018. Porto Alegre, RS. Brasil

The Periódico Tchê Química (ISSN: 1806-0374; 2179-0302) is an open-access journal since 2004. Journal DOI: 10.52571/PTQ. http://www.tchequimica.com.

The Periódico Tchê Quimica (ISSN: 1806-0374; 2179-0302) is an oper
This text was introduced in this file in 2021 for compliance reasons.
(O) The Author(s)

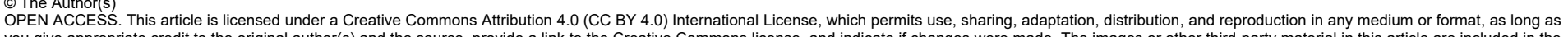
you give appropriate credit to the original author(s) and the source, provide a link to the Creative Commons license, and indicate if changes were made. The images or other third-party material in this article are included in the or exceeds the permitted use, you will need to obtain permission directly from the copyright holder. To view a copy of this license, visit http://creativecommons.org/licenses/by/4.0/. 